Electron collection and thermionic emission from a spherical dust grain in the spacecharge limited regime

A. Autricque, S. A. Khrapak, L. Couëdel, N. Fedorczak, C. Arnas, J.-M. Layet, and C. Grisolia

Citation: Physics of Plasmas 25, 063701 (2018); doi: 10.1063/1.5032153

View online: https://doi.org/10.1063/1.5032153

View Table of Contents: http://aip.scitation.org/toc/php/25/6

Published by the American Institute of Physics

\section{PHYSICS TODAY}

WHITEPAPERS
MANAGER'S GUIDE

Accelerate R\&D with Multiphysics Simulation
READ NOW

PRESENTED BY $\checkmark \subset \bigcirc M S O L$ 


\title{
Electron collection and thermionic emission from a spherical dust grain in the space-charge limited regime
}

\author{
A. Autricque, ${ }^{1, a)}$ S. A. Khrapak, ${ }^{2,3}$ L. Couëdel,,${ }^{4,5}$ N. Fedorczak, ${ }^{1}$ C. Arnas, ${ }^{5}$ J.-M. Layet,,${ }^{5,6}$ \\ and C. Grisolia ${ }^{1,6}$ \\ ${ }^{1}$ CEA, IRFM, F-13108 Saint-Paul-Lez-Durance, France \\ ${ }^{2}$ Institut für Materialphysik im Weltraum, Deutsches Zentrum für Luft- und Raumfahrt (DLR), 82234 Weßling, \\ Germany \\ ${ }^{3}$ Joint Institute for High Temperatures, Russian Academy of Sciences, 125412 Moscow, Russia \\ ${ }^{4}$ Physics and Engineering Physics, University of Saskatchewan, Saskatoon, Saskatchewan S7N 5E2, Canada \\ ${ }^{5}$ Université Aix-Marseille, CNRS, UMR 7345, PIIM, 13397 Marseille Cédex 20, France \\ ${ }^{6}$ National Research Nuclear University “MEPhI," Moscow 115409, Russia
}

(Received 3 April 2018; accepted 24 May 2018; published online 8 June 2018)

The collection and emission of electrons from a spherical body in the Space-Charge Limited (SCL) regime are investigated. When a Virtual Cathode (VC) in the potential profile around the body is present, the barrier in the effective potential energy of electrons is assumed to be located near the position of the minimum of the VC potential, for both collected and emitted electrons. This assumption is confirmed to be reasonable in the case of a double Yukawa potential profile and allows the SCL cross-section for electron collection and the emitted electron's trapped-passing boundary to be written in a simple way. An expression for the collection current for Maxwellian electrons is derived and is shown to recover the classical Orbital Motion Limited (OML) theory when the $\mathrm{VC}$ vanishes. Using the same assumptions, an expression for the thermionic emission current in the SCL regime is also obtained and comparisons with the $\mathrm{OML}^{+}$theory are made. Finally, an expression for the dust electric charge in the SCL regime is derived and shown to give drastically different results when compared to the commonly used formula (obtained from a Yukawa potential profile). Consequences in the framework of dust in tokamak plasmas are discussed. Published by AIP Publishing. https://doi.org/10.1063/1.5032153

\section{INTRODUCTION}

Dust grains have been observed in tokamaks for several decades. They are created through various processes in connection with plasma-wall interactions and will be a critical issue for future fusion devices such as ITER. ${ }^{1}$ In addition, the large amounts of impurities released by a dust grain subjected to a high erosion rate can lead to a reduced plasma performance by means of radiative losses. Impurities can also trigger plasma instabilities up to disruption.

When a small body, such as a dust grain or a probe is immersed in a plasma, it collects and emits charged particles. Due to higher electron mobility, the surface potential is usually negative and the body is negatively charged. This is true as long as the electron emission remains low. The two main electron emission processes relevant for dust in tokamak plasmas are secondary electron emission and thermionic emission (THE), ${ }^{2}$ the latter being the most important at high dust surface temperature. If the electron emission yield is high enough, a potential well, or virtual cathode (VC), forms in the sheath around it. In this so-called Space-Charge Limited (SCL) regime, the surface potential is increased and the body can become positively charged. ${ }^{3-5}$ The presence of a VC is not taken into account by the Orbital Motion Limited (OML) theory, ${ }^{6-8}$ which is commonly used to model dust/plasma interactions. ${ }^{9-13}$

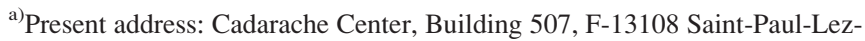
Durance, France. Electronic mail: adrien.autricque@cea.fr
}

The VC acts as a potential barrier for electrons, repelling the low-energy tail of their energy distribution function and thereby reducing both collected and emitted electron fluxes. The depth of the VC is of the order of the energy of the emitted electrons, which is equal to the dust surface temperature $T_{d}$ [in the case of Tungsten $(\mathrm{W}), T_{d} \sim 0.01-0.5 \mathrm{eV}$ ] if THE is the dominant emission process. Typical tokamak ScrapeOff Layer (SOL) plasma electrons usually have a higher temperature $\left(T_{e} \gtrsim T_{d}\right)$, meaning that the $\mathrm{VC}$ is expected to have a more important effect on emitted electrons than on primaries. The electron flux reduction affects the dust floating potential, electric charge, heating (therefore, lifetime), and transport in the vacuum vessel. Thus, it is crucial to assess electron collection and emission in the SCL regime.

Electron collection and emission by an electron emitting surface have been extensively studied, ${ }^{4,14}$ especially in the case of emissive probes. ${ }^{18}$ Yet, the emitting surface is usually assumed to be planar, which might be acceptable for millimeter probes in cold plasmas, but not in the case of dust grains where the orbital motion of charged particles plays a crucial role. Another model by Fruchtman et al. accounts for the probe curvature but considers cold ions and emitted electrons. ${ }^{19}$ In the case of dust grains, the electron emission reduction in the presence of a VC has been studied in the socalled $\mathrm{OML}^{+}$theory. ${ }^{20,21}$ An expression for the THE current is proposed and shows good agreement with Particle In Cell (PIC) simulations. It differs from the classical OML expression when the dust surface potential $\phi_{d}$ exceeds a critical 
value $\phi_{d}^{*}$. In order to use the $\mathrm{OML}^{+}$, it is required to solve $a$ priori the Poisson equation for various dust sizes and temperatures in order to obtain tables of $\phi_{d}^{*}$ that can be interpolated at each time step in a dust transport code. ${ }^{9-13}$ It also presents the limitation of considering the reduction of the emitted electron flux only, while the primary electron current is also expected to be significantly reduced in plasmas where the background temperature is of the order of the dust temperature. It was also recently demonstrated that an inverse sheath structure can appear near planar surfaces with electron emission yields exceeding unity and when collisions are important. $^{22-24}$ Again, orbital motion effects are not taken into account. Moreover, since a dust grain potential satisfies the floating condition, its electron emission yield remains below unity.

The dust electric charge is usually calculated using a formula obtained by assuming a monotonic Yukawa potential profile around the spherical body. ${ }^{25}$ As pointed out in Ref. 20, this expression is no longer valid in the SCL regime, when the body is positively charged regardless of the sign of the surface potential. Thus, a new expression for the dust charge in the SCL regime must be found.

In this paper, we propose new expressions for the electron collection and emission currents by a spherical body in the SCL regime. We focus on the case where collisions in the sheath can be neglected to avoid inverse sheath effects.

This paper is organized as follows: In Sec. II, the electron collection cross-section is determined and the expression for the current is derived. In Sec. III, an expression for the THE current is proposed and compared with well-known OML and $\mathrm{OML}^{+}$results. In Sec. IV, different methods for estimating the location and depth of the VC available in the literature are discussed. In Sec. V, an expression for the dust electric charge in the SCL regime is proposed.

\section{ELECTRON COLLECTION IN THE SPACE-CHARGE LIMITED REGIME}

\section{A. Determination of the cross-section for collection}

In the vicinity of an electrically charged spherical body, another charged particle is subjected to several interactions, primarily electromagnetic forces and collisions with other plasma particles. It is commonly assumed that the particle evolves in a central force field, meaning that the electric force is dominant. In other words, we assume that the trajectory of the particle in the sheath around the body is collisionless, and magnetic field effects are neglected. Thus, the results presented herein hold for $r_{d} \ll \lambda_{e, i}, \rho_{\mathrm{Le}, \mathrm{i}}$, where $r_{d}$ is the radius of the body, $\lambda_{e, i}$ is the electron (ion) mean free path, and $\rho_{\mathrm{Le}, \mathrm{i}}$ is the electron (ion) Larmor radius. The term "charged particle" can refer to both electrons and ions.

It follows from the conservation of the total energy and angular momentum that the behavior of the charged particle in a central force field is determined by the effective potential energy (normalized to the initial kinetic energy)

$$
U_{\text {eff }}(\rho, r)=\left(\frac{\rho}{r}\right)^{2}+\frac{2 U(r)}{m v^{2}},
$$

where $\rho, r, m$, and $v$ are the particle impact parameter, radial position, mass, and velocity at infinity, respectively. ${ }^{26,27}$ $U(r)=z e \phi(r)$ is the potential energy, where $z$ is the particle charge number, $e$ is the elementary charge, and $\phi$ is the electric potential. The particle motion is restricted to the area where $U_{\text {eff }}(\rho, r) \leq 1$. Figure 1 shows a schematic view of the problem.

Our goal is to find the critical impact parameter $\rho_{c}(v)$ above which a particle of velocity $v$ cannot be collected by the body. The impact parameter $\rho_{c}$ is found by solving $U_{\text {eff }}\left(\rho, r_{d}\right)=1$. Then, the cross-section for collection will be defined by $\sigma=\pi \rho_{c}^{2}$.

In general, the problem is complicated because the equation $U_{\text {eff }}=1$ can have several solutions when the particle is attracted to the body. The OML theory makes the assumption that there are no barriers in $U_{\text {eff }}$ and that $\phi$ is monotonic. In this case, the equation $U_{\mathrm{eff}}\left(\rho, r_{d}\right)=1$ (which is equivalent to writing the conservation of energy and angular momentum between the sheath entrance and the body surface) has a unique solution corresponding to the OML critical impact parameter $\rho_{c}^{\mathrm{OML}}$. It can be easily determined from the dust radius $r_{d}$ and potential $\phi_{d}$

$$
\rho_{c}^{\mathrm{OML}}(v)= \begin{cases}r_{d} \sqrt{1-\frac{2 z e \phi_{d}}{m v^{2}}} & \text { if } v \geq \sqrt{\frac{2 z e \phi_{d}}{m}} \\ 0 & \text { if } v<\sqrt{\frac{2 z e \phi_{d}}{m}} .\end{cases}
$$

Note that the second line in Eq. (2) should be taken into account only when $z$ and $\phi_{d}$ are of the same sign. In this case, the body repels the charged particle (because $\phi$ is monotonic) and there exists a critical kinetic energy below which the body cannot be reached. It has been demonstrated (in the case of a Yukawa potential) that the OML assumptions are justified when the condition $r_{d} \ll \lambda_{D}$ holds $\left(\lambda_{D}\right.$ is the Debye length). ${ }^{27}$

In the SCL regime, the non-monotonicity of $\phi$ may induce the emergence of barriers in $U_{\text {eff }}$ for incoming particles, which makes the situation significantly more complex. Barriers in $U_{\text {eff }}$ is a well-known problem that has

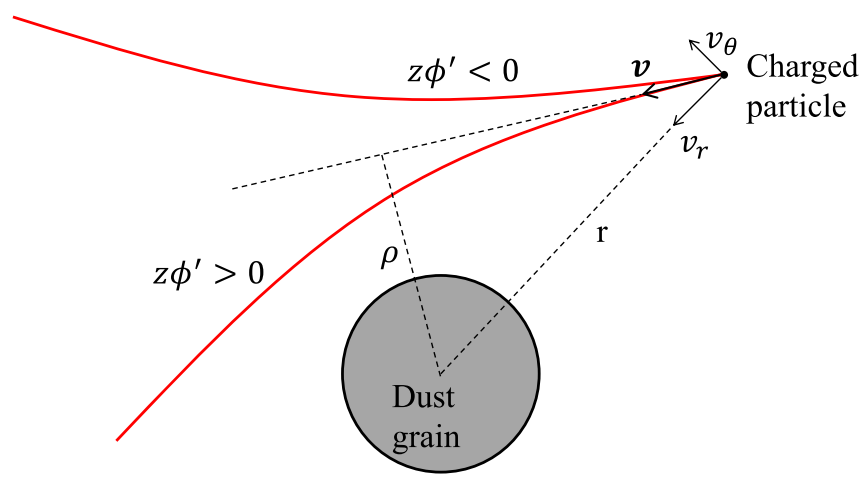

FIG. 1. Schematic view of the parameters required to describe the behavior of a charged particle in the central force field generated by a spherical body (dust grain). At a given radial position $r$, the particle can be either attracted or repelled depending on the sign of $z \phi^{\prime}(r)$. 
been extensively studied in the case of incoming ions in monotonic potential profiles under the name of the absorption radius effect. ${ }^{7,26,28-30}$ In the case of a non-monotonic potential profile, barriers are expected to emerge for both electrons and positive ions. Figure 2 shows profiles of $U_{\text {eff }}$ for electrons computed with a double Yukawa potential [see Eq. (5)].

The largest solution to the equation $U_{\text {eff }}(\rho, r)=1$ corresponds to the actual critical impact parameter. Barriers, located at $r_{M}$ (which depends on the particle velocity $v$ ), are found by solving $U_{\text {eff }}=1$ and $d U_{\text {eff }} / d r=0$, which is equivalent to

$$
r_{M}^{3} \frac{d U}{d r}\left(r_{M}\right)=m v^{2} \rho_{*}^{2},
$$

where $\rho_{*}$ is named the transitional impact parameter and is defined by

$$
\rho_{*}(v)=r_{M} \sqrt{1-\frac{2 z e \phi\left(r_{M}\right)}{m v^{2}}} .
$$

It is obvious that knowledge of the full potential profile is required to find the barrier. In the following, we study the special case of a double Yukawa profile:

$$
\begin{aligned}
\phi(r)= & \left(\phi_{d}+\phi_{0}\right) \frac{r_{d}}{r} \exp \left(\xi \frac{r_{d}-r}{\lambda}\right) \\
& -\phi_{0} \frac{r_{d}}{r} \exp \left(\frac{r_{d}-r}{\lambda}\right)
\end{aligned}
$$

where $\phi_{0}, \lambda$, and $\xi$ are parameters. ${ }^{31}$ It was shown that this expression can fit accurately results from the exact Orbital Motion (OM) theory. ${ }^{32}$ Note that $\lambda$ is the characteristic screening length of the second term in Eq. (5), which is the one that decays the slowest (since we use $\xi>1$ ). Hence, $\lambda$ should correspond to the plasma Debye length. Two

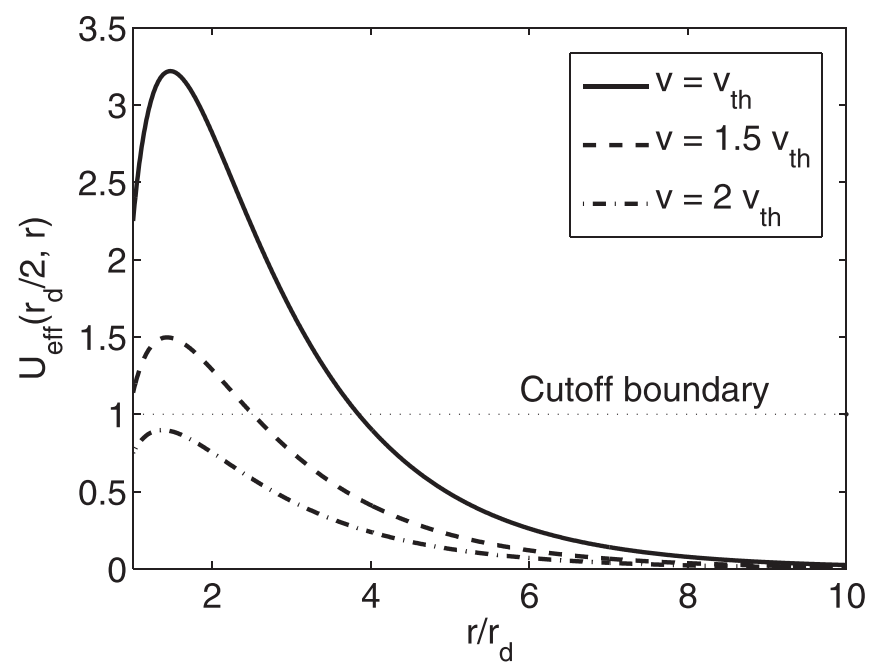

FIG. 2. Effective potential energy $U_{\text {eff }}$ of incoming electrons $(z=-1)$ versus the distance $r$ for $\rho=r_{d} / 2$ and different particle velocities. The largest solution of the equation $U_{\text {eff }}=1$ corresponds to the distance of the closest approach, since particles are not allowed to exist in the region $U_{\text {eff }}>1$. A double Yukawa potential, Eq. (5), is used, with $e \phi_{d} / T_{e}=-1, e \phi_{0} / T_{e}=10$, $\lambda / r_{d}=2$ and $\xi=2$. examples of double Yukawa profiles are plotted in Fig. 3 for $\lambda=2 r_{d}, \xi=2, e \phi_{d} / T_{e}=-1, e \phi_{0} / T_{e}=10$ (a) and $e \phi_{d} / T_{e}$ $=0.5, e \phi_{0} / T_{e}=5$ (b). In the following, $T$ designates the temperature of the plasma species, with a subscript $e(i)$ for electrons (ions) when required.

The position of the barrier, $r_{M}$, is found by solving Eq. (3), which is equivalent to (after some straightforward algebra) solving the transcendental equation

$$
\begin{aligned}
& \left(1+\frac{\phi_{d}}{\phi_{0}}\right)\left(1-\xi \frac{r_{M}}{\lambda}\right) \exp \left(\xi \frac{r_{d}-r_{M}}{\lambda}\right) \\
& -\left(1-\frac{r_{M}}{\lambda}\right) \exp \left(\frac{r_{d}-r_{M}}{\lambda}\right)=\frac{r_{M}}{r_{d}} \frac{m v^{2}}{z e \phi_{0}} .
\end{aligned}
$$

On the other hand, the location of the minimum of $\phi$ (due to the VC), named $r_{\min }$, is found by solving $\phi^{\prime}\left(r_{\min }\right)$ $=0$, i.e.,

$$
\begin{gathered}
\left(1+\frac{\phi_{d}}{\phi_{0}}\right)\left(1+\xi \frac{r_{\text {min }}}{\lambda}\right) \exp \left(\xi \frac{r_{d}-r_{\text {min }}}{\lambda}\right) \\
=\left(1+\frac{r_{\text {min }}}{\lambda}\right) \exp \left(\frac{r_{d}-r_{\text {min }}}{\lambda}\right) .
\end{gathered}
$$

A fundamental difference between $r_{M}$ and $r_{\min }$ is that the latter is a constant defined by the potential profile, while the first depends on the velocity of the charged particle $v$. The important assumption we bring here is that, in the case of electrons $(z=-1)$, we can approximate the location of the barrier in the effective potential energy by the VC itself, i.e., $r_{M} \approx r_{\min }$. If verified, the currents can be calculated for the known location and depth of the VC. The exact values of $r_{M}$ for electrons were calculated for various shapes of the double Yukawa profile, varying $\phi_{d}, \phi_{0}, \lambda$, and $\xi$. The two extreme cases, corresponding to the largest deviation between $r_{M}$ and $r_{\text {min }}$, are plotted in Fig. 4.

For all the cases tested, the ratio $r_{M} / r_{\min }$ remains in the range of $0.5-3$. Keeping in mind that electrons are Maxwellian distributed, the most probable velocity will be $v_{\text {th }} \sqrt{2}\left(v_{\text {th }}=\sqrt{T / m}\right.$ is the thermal velocity), where $r_{M} / r_{\text {min }}$ is very close to unity. Thus, we conclude that this approximation is reasonable.

We now define the so-called VC impact parameter $\rho_{\mathrm{VC}}$ in the same way as $\rho_{*}$ but replacing $r_{M}$ with $r_{\min }$, i.e.,

$$
\rho_{\mathrm{VC}}(v)=r_{\min } \sqrt{1-\frac{2 z e \phi_{\min }}{m v^{2}}},
$$

where $\phi_{\min }=\phi\left(r_{\min }\right)$. Figures 3(c) and 3(d) show $\rho_{*}$ and $\rho_{\mathrm{VC}}$ for electrons $(z=-1)$ plotted versus the particle velocity normalized to $v_{\text {th }}$ for the potential profiles plotted in Figs. 3(a) and 3(b), respectively. Both impact parameters are actually very close in the interval of velocity where they are both below the OML critical impact parameter $\rho_{c}^{\mathrm{OML}}$. When a barrier exists in $U_{\text {eff }}$, particles with $\rho(v) \geq \rho_{*}(v)$ cannot be collected, so the critical impact parameter for collection departs from the OML one and saturates at $\rho_{*}(v)$ when $\rho_{c}^{\mathrm{OML}}(v)$ $\geq \rho_{*}(v)$. Thus, the critical impact parameter for collection in the SCL regime is 
(a)

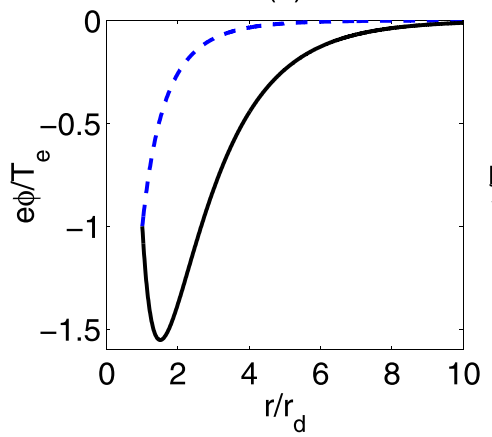

(c)
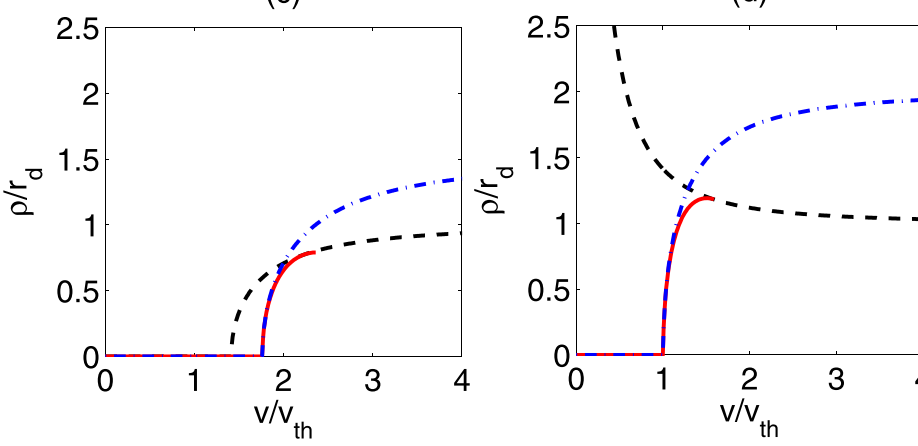

(d)

$$
\rho_{c}(v)= \begin{cases}r_{d} \sqrt{1-\frac{2 z e \phi_{d}}{m v^{2}}} & \text { if } r_{d} \sqrt{1-\frac{2 z e \phi_{d}}{m v^{2}}} \leq \rho_{*}(v) \\ \rho_{*} & \text { if } r_{d} \sqrt{1-\frac{2 z e \phi_{d}}{m v^{2}}}>\rho_{*}(v) \\ 0 & \text { if } v \leq \sqrt{\frac{2 z e \phi\left(r_{M}\right)}{m}}\end{cases}
$$

Since $\rho_{*}$ and $\rho_{\mathrm{VC}}$ are actually close [see Figs. 3(c) and 3(d)], we can approximate $\rho_{c}$ in the SCL regime by substituting $\rho_{*}$ by $\rho_{\mathrm{VC}}$ in Eq. (9). We find

$$
\rho_{c}^{\mathrm{SCL}}(v)= \begin{cases}r_{d} \sqrt{1-\frac{2 z e \phi_{d}}{m v^{2}}} & \text { if } v>v_{m} \\ r_{\min } \sqrt{1-\frac{2 z e \phi_{\min }}{m v^{2}}} & \text { if } v_{c}<v \leq v_{m} \\ 0 & \text { if } v \leq v_{c},\end{cases}
$$

where

$$
v_{c}=\sqrt{\frac{2 z e \phi_{\min }}{m}} \text { and } v_{m}=\sqrt{\frac{2 z e}{m}\left(\frac{\chi \phi_{d}-\phi_{\min }}{\chi-1}\right)},
$$

and $\chi=\left(r_{d} / r_{\text {min }}\right)^{2}$. Again, the last lines in Eqs. (9) and (10) should be taken into account only when $z$ and $\phi\left(r_{M}\right)$ (or $\left.\phi_{\min }\right)$ are of the same sign (i.e., for ions). This impact parameter can be understood as follows: (i) if $v \leq v_{c}$, the incoming electron does not have a sufficient kinetic energy to overcome the VC and cannot be collected; (ii) if $v_{c}<v \leq v_{m}$, a barrier in the effective potential energy exists but particles have a sufficiently high kinetic energy to pass the VC and are collected; (iii) if $v>v_{m}$, particles have such a high kinetic energy that they do not see the $\mathrm{VC}$ and the critical impact parameter is identical to the OML one.
FIG. 3. Double Yukawa potential profile for $\lambda=2 r_{d}, \xi=2$, e $\phi_{d} / T_{e}=-1$, $e \phi_{0} / T_{e}=10$ (a) and $e \phi_{d} / T_{e}=0.5$, $e \phi_{0} / T_{e}=5$ (b). The VCs are located at $r_{\text {min }} \approx 1.5 r_{d}$ (a) and $r_{\min } \approx 2 r_{d}$ (b). OML critical impact parameter, transitional impact parameter, and $\rho_{\mathrm{VC}}$ against the particle velocity normalized to the thermal velocity, in the case of electrons (c) and (d). (c) and (d) correspond to the potential profiles plotted in (a) and (b), respectively.

We note that when $\phi_{\min } \rightarrow \phi_{d}, v_{m} \rightarrow v_{c}$, which leads to the disappearance of the second line in Eq. (10). In this case, we recover the classic OML impact parameter.

The final cross-section for collection is $\sigma=\pi \rho_{c}^{2}$. The slight overestimation of the cross-section obtained with $\rho_{\mathrm{VC}}$ instead of $\rho_{*}$ is due to the fact that $r_{M}<r_{\text {min }}$ in this regime of velocities. The error in the interval $v_{c} \leq v \leq v_{m}$ corresponds to the area where $\rho_{*} \leq \rho \leq \rho_{\mathrm{VC}}, \rho_{c}^{\mathrm{OML}}$ in Figs. 3(c) and 3(d) and reaches $\sim 30 \%$ in this case.

In all calculations performed in the case of ions using the double Yukawa profile, we found the barrier to be located inside the grain, $r_{M}<r_{d}$, meaning that the exact cross-section for collection is equal to $\sigma_{\mathrm{OML}}$ with Eq. (2). In this case, the ion current collected by the dust grain is given

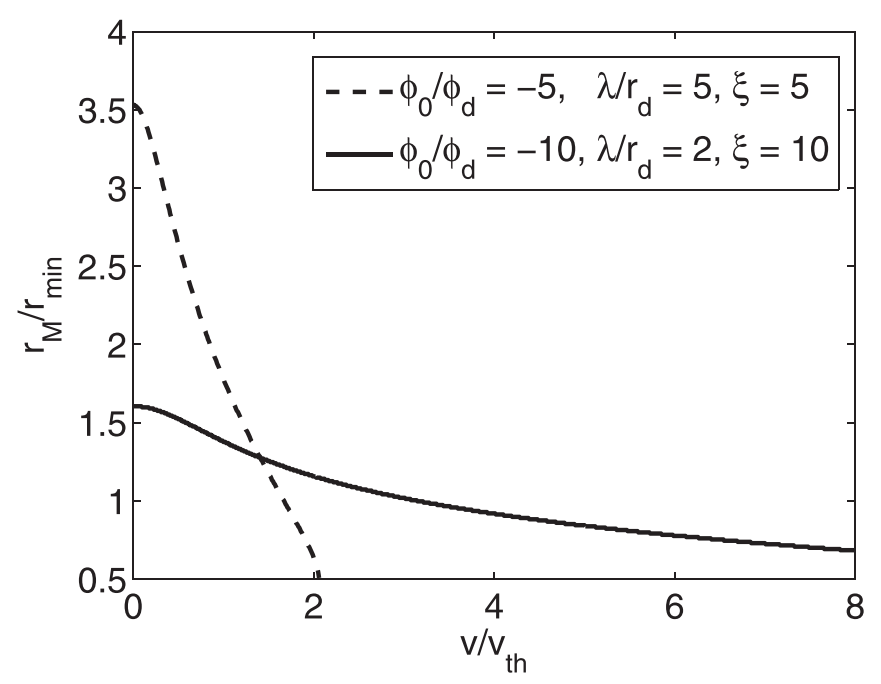

FIG. 4. Ratio of the location of the barrier in $U_{\text {eff }}$ from Eq. (6) to the location of the $\mathrm{VC}$ from Eq. (7) against the particle velocity $v . r_{M} / r_{\min }$ remains in the range $0.5-3$. We used $e \phi_{d} / T_{e}=-1$. 
by the well-known OML expression. In the following, we will focus on electrons and approximate the exact critical impact parameter $\rho_{c}$ with $\rho_{c}^{\mathrm{SCL}}$ since the $\mathrm{VC}$ is easier to compute than the barrier in the effective potential.

\section{B. Electron collection current}

The electron current density is obtained by integrating the collection cross-section with the velocity distribution function $f_{e}$ of the species

$$
J_{e}=\frac{e}{4 \pi r_{d}^{2}} \int v f_{e}(v) \sigma(v) d^{3} v
$$

where $d^{3} v=4 \pi v^{2} d v$ and $f_{e}$ is commonly assumed to be a Maxwellian

$$
f_{e}(v)=n_{e}\left(\frac{m_{e}}{2 \pi T_{e}}\right)^{3 / 2} \exp \left(-\frac{m_{e} v^{2}}{2 T_{e}}\right),
$$

where $n_{e}$ is the background electron density. When substituting $\sigma=\pi \rho_{c}^{\mathrm{SCL}}{ }^{2}$ in Eq. (12) and normalizing to the random current $J^{0}=\frac{1}{4} e n \sqrt{8 T / \pi m}$, we obtain, using the change of variable $v=u \sqrt{2 T_{e} / m_{e}}$

$$
\begin{aligned}
\frac{J_{e}}{J_{e}^{0}}= & \frac{2}{\chi} \int_{u_{c}}^{u_{m}}\left(u^{3}+u \frac{e \phi_{\min }}{T_{e}}\right) e^{-u^{2}} d u \\
& +2 \int_{u_{m}}^{\infty}\left(u^{3}+u \frac{e \phi_{d}}{T_{e}}\right) e^{-u^{2}} d u,
\end{aligned}
$$

where

$$
u_{c}=\sqrt{-\frac{e \phi_{\min }}{T_{e}}}
$$

and

$$
u_{m}=\sqrt{\frac{1}{1-\chi}\left[\frac{e\left(\chi \phi_{d}-\phi_{\min }\right)}{T_{e}}\right]} .
$$

After integration, we obtain the final expression for the electron current in the SCL regime

$$
\frac{J_{e}}{J_{e}^{0}}=\frac{1}{\chi} \exp \left(\frac{e \phi_{\min }}{T_{e}}\right)\left[1+(\chi-1) e^{\tilde{\varphi}}\right],
$$

where

$$
\tilde{\varphi}=\frac{\chi}{1-\chi} \frac{e\left(\phi_{\min }-\phi_{d}\right)}{T_{e}} .
$$

This expression is also valid when the VC vanishes, in which case the OML expression is recovered. Indeed, the disappearance of the VC is obtained by $r_{\min }=r_{d}$ and $\phi_{\min }=\phi_{d}$ if $\phi_{d} \leq 0$, and by $r_{\min } \rightarrow \infty$ and $\phi_{\min }=0$ if $\phi_{d}>0$.

Moreover, this more general expression allows us to extend the validity domain of the theory to larger collectors, since the assumption $r_{d} \ll \lambda_{D}$ made in the OML that ensures the negligible role of barriers in $U_{\text {eff }}$ can be dropped.
However, the collisionless and unmagnetized plasma assumptions still require $r_{d} \ll \lambda_{e, i}, \rho_{\mathrm{Le}, \mathrm{i}}$. Hence, the maximum body size that can be used depends on the plasma background temperature, density, and the magnetic field (if present).

\section{THERMIONIC EMISSION IN THE SPACE-CHARGE LIMITED REGIME}

\section{A. Generalities on thermionic emission from a spherical dust grain}

When heated up to high temperatures, dust grains and emissive probes $^{18}$ emit electrons through the THE effect. The THE current density from an uncharged body is given by the Richardson-Dushman formula ${ }^{33}$

$$
J_{\text {th }}^{0}=e \frac{4 \pi m_{e} T_{d}^{2}}{h^{3}} \exp \left(-\frac{W_{f}}{T_{d}}\right),
$$

where $T_{d}$ is the body surface temperature, $h$ is the Planck constant, and $W_{f}$ is the material work function. In the OML framework, the sheath potential profile is assumed to be monotonic. In this case, the current is given by Eq. (19) when $\phi_{d} \leq 0$, since all emitted electrons are repelled from the body and escape the sheath. When $\phi_{d}>0$, part of the emitted electrons (corresponding to the low energy tail of their distribution function) is attracted back, forming a return current that effectively reduces electron emission. Electrons that manage to escape the sheath are named passing electrons, while those that return to the body are named trapped.

The THE current accounting for the potential drop is obtained by integrating the velocity distribution function of THE electrons, named $f_{\text {th }}$, over the passing electron population

$$
J_{\text {th }}=e \int_{\text {pas. }} v f_{\text {th }}(v) d^{2} v,
$$

where $d^{2} v=2 \pi v d v$. In the following, we assume the THE electrons to follow a Maxwellian distribution associated with the temperature $T_{d}$. The velocity vector distribution function is written as

$$
f_{\text {th }}(v)=v\left(\frac{m_{e}}{h}\right)^{3} \exp \left(-\frac{m_{e} v^{2}}{2 T_{d}}-\frac{W_{f}}{T_{d}}\right) .
$$

This distribution ensures that Eq. (19) is recovered when integrated over the whole velocity space.

\section{B. OML case}

The distinction between trapped and passing electrons is named Trapped-Passing Boundary (TPB) and is given by the energy conservation. In the OML case (monotonic potential profile), the TPB is, when $\phi_{d}>0,{ }^{34}$

$$
v_{r}^{2}+v_{\theta}^{2}=\frac{2 e}{m_{e}} \phi_{d} .
$$

The OML TPB is a circle shown in the velocity space in Fig. 5, where the trapped population is located inside the dotted area.

The OML THE current $J_{\text {th }}^{\mathrm{OML}}$ is obtained by integrating in the velocity space outside of the TPB 


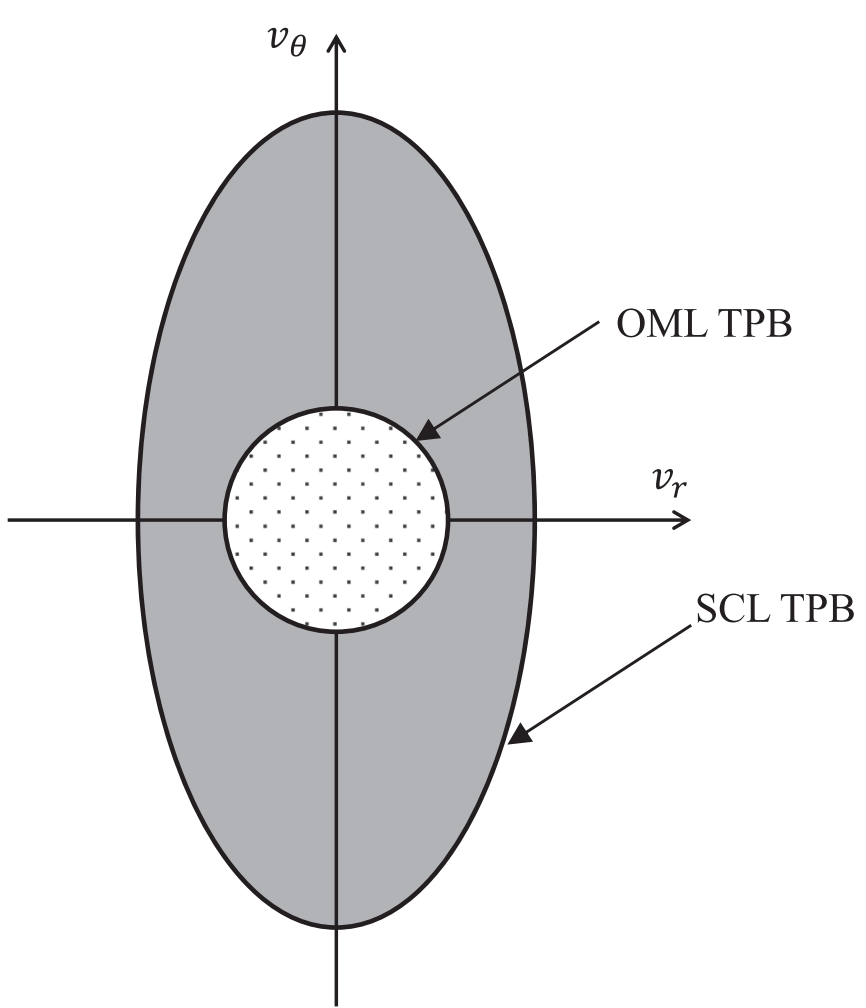

FIG. 5. Integration domains for the determination of the THE currents, delimited by the TPBs for the OML and SCL cases.

$$
\frac{J_{\mathrm{th}}^{\mathrm{OML}}}{J_{\mathrm{th}}^{0}}=\frac{1}{\pi} \int_{u_{r}^{2}+u_{\theta}^{2} \geq e \phi_{d} / T_{d}} u^{2} e^{-u^{2}} d^{2} u .
$$

This integral can be calculated by switching to polar coordinates in the velocity space. We obtain the well-known expression for positively charged spherical bodies ${ }^{34}$

$$
\frac{J_{\text {th }}^{\mathrm{OML}}}{J_{\mathrm{th}}^{0}}=\left(1+\frac{e \phi_{d}}{T_{d}}\right) \exp \left(-\frac{e \phi_{d}}{T_{d}}\right) .
$$

\section{SCL case}

In the SCL regime, the emitted electrons can experience potential barriers in the effective potential energy, identically to collected electrons. The TPB in the SCL regime is not easily defined. Similarly to Sec. II A and Ref. 20, we assume that the barrier in the effective potential energy due to the presence of the $\mathrm{VC}$ is located at the minimum of the $\mathrm{VC}$, i.e., $r_{\text {min }}$. This means that the SCL TPB can be approximated by

$$
v_{r}^{2}+(1-\chi) v_{\theta}^{2}=v_{p}^{2},
$$

where we introduce $v_{p}=\sqrt{2 e\left(\phi_{d}-\phi_{\min }\right) / m_{e}}$. In the velocity space, this contour is an ellipse elongated along the $v_{\theta}$ axis and is represented in Fig. 5. The emission current is obtained by integrating outside of the TPB. We use the following change of variables:

$$
\begin{aligned}
& v_{r}=u \sqrt{\frac{2 T_{d}}{m_{e}}} \cos \alpha, \\
& v_{\theta}=u \sqrt{\frac{2 T_{d}}{m_{e}}} \frac{\sin \alpha}{\sqrt{1-\chi}} .
\end{aligned}
$$

After integration by parts, Eq. (20) becomes

$$
\frac{J_{\mathrm{th}}^{\mathrm{SCL}}}{J_{\mathrm{th}}^{0}}=1-\frac{K_{1}}{\sqrt{1-\chi}}+\frac{1}{\sqrt{1-\chi}}\left(K_{2} u_{p}^{2}+K_{3}\right) e^{-u_{p}^{2}},
$$

where $u_{p}=\sqrt{e\left(\phi_{d}-\phi_{\min }\right) / T_{d}}$ and

$$
\begin{aligned}
& K_{1}=\frac{2}{\pi} \int_{0}^{\pi / 2} \frac{d \alpha}{1+\frac{\chi}{1-\chi} \sin ^{2} \alpha}, \\
& K_{2}=\frac{2}{\pi} \int_{0}^{\pi / 2} \exp \left(-u_{p}^{2} \frac{\chi}{1-\chi} \sin ^{2} \alpha\right) d \alpha \\
& K_{3}=\frac{2}{\pi} \int_{0}^{\pi / 2} \frac{1}{1+\frac{\chi}{1-\chi} \sin ^{2} \alpha} \exp \left(-u_{p}^{2} \frac{\chi}{1-\chi} \sin ^{2} \alpha\right) d \alpha .
\end{aligned}
$$

One can find that

$$
\begin{aligned}
& K_{1}=\sqrt{1-\chi} \\
& K_{2}=\exp \left(-\frac{u_{p}^{2}}{2} \frac{\chi}{1-\chi}\right) I_{0}\left(\frac{u_{p}^{2}}{2} \frac{\chi}{1-\chi}\right), \\
& K_{3}=g\left(u_{p}, u_{p} \sqrt{\frac{\chi}{1-\chi}}\right),
\end{aligned}
$$

where $I_{0}$ is the modified Bessel function of the first kind and $g$ is a function defined as

$$
g(x, y)=\frac{2}{\pi} \frac{1}{y} \int_{0}^{y} \frac{1}{1+(t / x)^{2}} \frac{e^{-t^{2}}}{\sqrt{1-(t / y)^{2}}} d t .
$$

Since the typical VC depth is of the order of the dust surface temperature, we expect $u_{p} \gtrsim 1$. In this case, $g\left(u_{p}, y\right)$ can be approximated by $f(y)=\exp \left(-y^{2} / 2\right) I_{0}\left(y^{2} / 2\right)$, as can be seen in Fig. 6. On this ground, the THE current can be expressed as

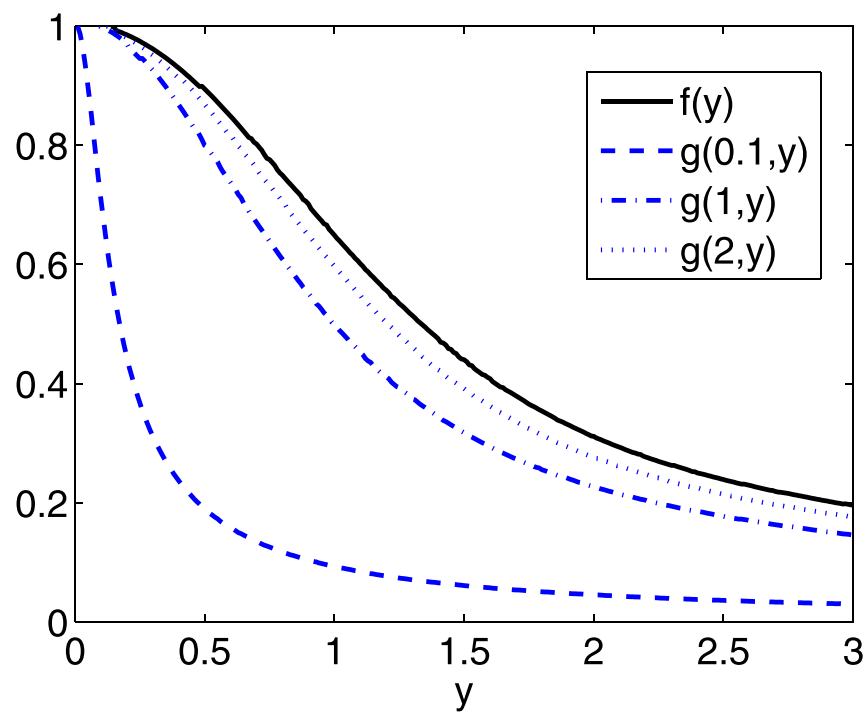

FIG. 6. $f$ and $g$ functions. $g\left(u_{p}, \cdot\right)$ can be approximated with $f$ for $u_{p} \gtrsim 1$. 
$\frac{J_{\mathrm{th}}^{\mathrm{SCL}}}{J_{\mathrm{th}}^{0}}=\frac{1}{\sqrt{1-\chi}}\left(1+u_{p}^{2}\right) e^{-u_{p}^{2}} \exp \left(-\frac{u_{p}^{2}}{2} \frac{\chi}{1-\chi}\right) I_{0}\left(\frac{u_{p}^{2}}{2} \frac{\chi}{1-\chi}\right)$.

We observe that, since $I_{0}(0)=1$, Eq. (31) recovers the expression proposed in the $\mathrm{OML}^{+}$theory for $\chi \ll 1$, i.e., when the $\mathrm{VC}$ is far from the grain. The expression from Eq. (31) is compared with exact calculations of Eq. (27) in Fig. 7 (a), where $J_{\text {th }}^{\mathrm{SCL}}$ is plotted for $e \phi_{d} / T_{e}=0, T_{d} / T_{e}=0.2$ and $\chi=1 / 4$ as a function of the VC depth $\phi_{\min }$.

The agreement is nearly perfect for large $\phi_{\min }$ because $u_{p} \gtrsim 1$. As $\phi_{\min } \rightarrow 0$, Eq. (31) leads to overestimations and the current calculated can exceed the saturation value. We conclude that the THE current in the SCL regime can be reasonably estimated by Eq. (31) with a forced saturation at $J_{\text {th }}^{0}$ when Eq. (31) gives $J_{\text {th }}^{\mathrm{SCL}}>J_{\text {th }}^{0}$.

The new expression for the THE current is then compared with the $\mathrm{OML}^{+}$theory. Identically to $\mathrm{OML}^{+}$, we assume that the VC appears when $\phi_{d}$ exceeds the critical value $\phi_{d}^{*}$ which depends on $T_{d} / T_{e}$ and $r_{d} / \lambda_{D}$. Once the VC is formed, we use reasonable values for its depth, i.e., $\phi_{\min }=\phi_{d}^{*}$ (see Fig. 2 from Ref. 20) and vary the VC position $\chi$. The currents are plotted in Fig. 7(b) for $r_{d}=\lambda_{D}$ and $T_{d} / T_{e}=0.2$, corresponding to $e \phi_{d}^{*} / T_{e}=-0.125$ according to $\mathrm{OML}^{+}$. It is confirmed that the new expression for the THE current in the SCL regime recovers the $\mathrm{OML}^{+}$when the $\mathrm{VC}$ is located far from the grain $(\chi \ll 1)$. When $\chi \sim 1$, the THE current is reduced. This can be understood using the SCL TPB: electrons are passing if $v^{2} \geq v_{p}^{2}+\chi v_{\theta}^{2}$, meaning that there are less passing electrons as $\chi \rightarrow 1$.

\section{DESCRIPTION OF THE VIRTUAL CATHODE}

Describing the $\mathrm{VC}$ is easier than the barriers in the effective potential energy, yet it is still not straightforward. To be perfectly accurate, one should solve the Poisson equation in the OM framework, as was done in Ref. 32. Since we are looking for a fast and simple way to estimate the currents in the SCL regime, we need a direct expression for $r_{\min }$ and $\phi_{\text {min }}$. In the following, we will use results from Ref. 32 that allow an estimate of the VC parameters within $15 \%$ accuracy when compared with exact calculations made with the OM theory. The transcendental equation for $\phi_{\min }$ is

$$
\begin{aligned}
\tilde{\phi}_{\text {min }}^{2}(1+\beta)= & \frac{\tilde{n}_{\text {th }}}{4}\left(\frac{r_{d}}{\lambda_{D}}\right)^{2}\left(1-\operatorname{erf} \sqrt{-\delta \tilde{\phi}_{\text {min }}}\right) \\
& \times\left(\exp \left(\tilde{\phi}_{\text {min }}\right)-\exp \left(-\beta \tilde{\phi}_{\text {min }}\right)\right. \\
& \left.+H \exp \left(\delta \tilde{\phi}_{\text {min }}\right)\right),
\end{aligned}
$$

where $\tilde{\phi}_{\min }=e \phi_{\min } / T_{e}, \beta=T_{e} / T_{i}, \delta=T_{e} / T_{d}$,

$$
\begin{aligned}
H^{-1}= & \frac{\left(1+\delta \tilde{\phi}_{\min }\right)}{\tilde{\phi}_{\min }(1+\beta)}\left(\operatorname{erf} \sqrt{-\delta \tilde{\phi}_{\min }}-1\right) \\
& -\frac{\exp \left(\delta \tilde{\phi}_{\min }\right) \sqrt{-\delta \tilde{\phi}_{\min } / \pi}}{\tilde{\phi}_{\min }(1+\beta)}
\end{aligned}
$$

and

$$
\tilde{n}_{\mathrm{th}}=\frac{J_{\mathrm{th}}^{0}}{2 J_{e}^{0}} \exp \left(-\frac{e \phi_{d}}{T_{d}}\right) .
$$

Then, the VC position is obtained with

$$
\chi=\frac{H}{\tilde{n}_{\mathrm{th}}} .
$$

Another argument states that the depth of the VC is of the order of the energy of emitted electrons that is, in the case of THE, the dust temperature $T_{d}{ }^{35}$ Finally, the $\mathrm{OML}^{+}$theory allows the estimation of the dust critical potential $\phi_{d}^{*}$ above which the VC appears. ${ }^{20}$ In Figs. 8(a) and 8(b) are plotted the $\mathrm{VC}$ parameters using $\mathrm{OM}$ results against the dust potential for a W dust grain with $r_{d}=1 \mu \mathrm{m}$ and $T_{d}=4500 \mathrm{~K}$ and for two different electron temperatures $(0.5$ and $2 \mathrm{eV})$. In both cases, $e \phi_{d}^{*} / T_{e} \approx-0.125$ according to $\mathrm{OML}^{+}$. This value differs from the estimations made in the figure, where the VC appears at $e \phi_{d} / T_{e}<-2$, according to the OM theory. Due to lack of any conclusive argument on which values to use, we opt for the $\mathrm{OM}$ results since more physics is included.

This link between $\phi_{d}$ and $\phi_{\min }$ allows the determination of the dependence of the OML and SCL currents on $\phi_{d}$, for a given set of plasma parameters. This presents an improvement to the $\mathrm{OML}^{+}$theory, where Poisson's equation had to be solved a priori for the value $\phi_{d}^{*}$ to be known.

In Figs. 8(c) and 8(d) are plotted the OML and SCL currents against the normalized body potential. We observe that (a)

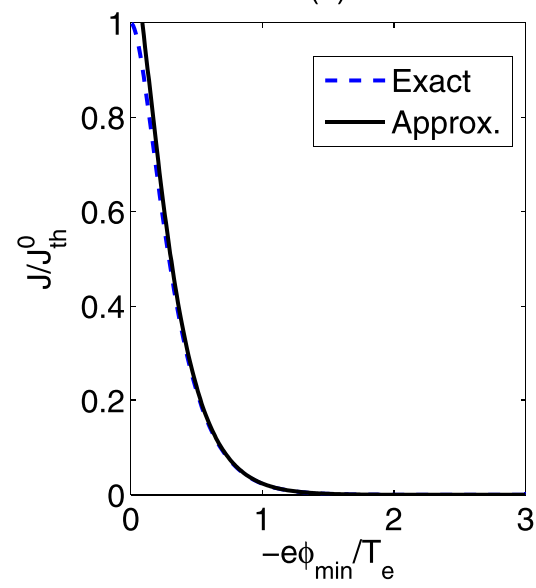

(b)

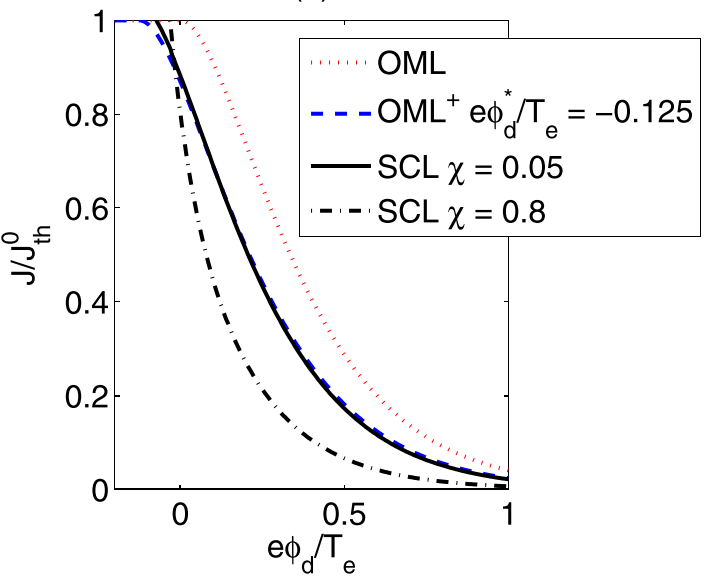

FIG. 7. (a) THE current from Eq. (27) (solid line) and Eq. (31) (dashed line). (b) THE currents from OML (dotted line), $\mathrm{OML}^{+}$with $e \phi_{d}^{*} / T_{e}=-0.125$ (dashed line), Eq. (31) with $\chi=0.05$ (solid line) and $\chi=0.8$ (dashed-dotted line). 
(a)

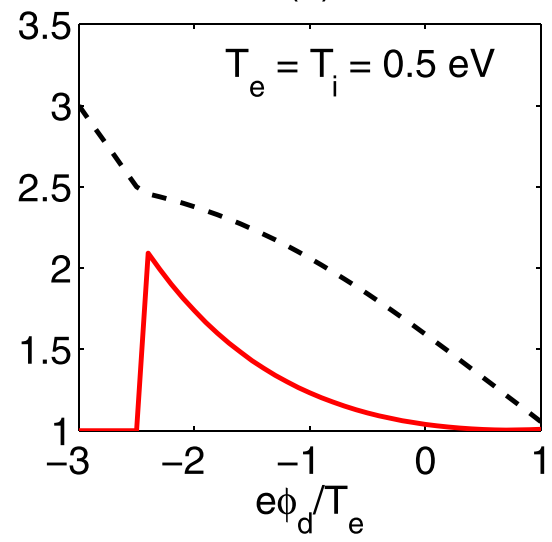

(c)

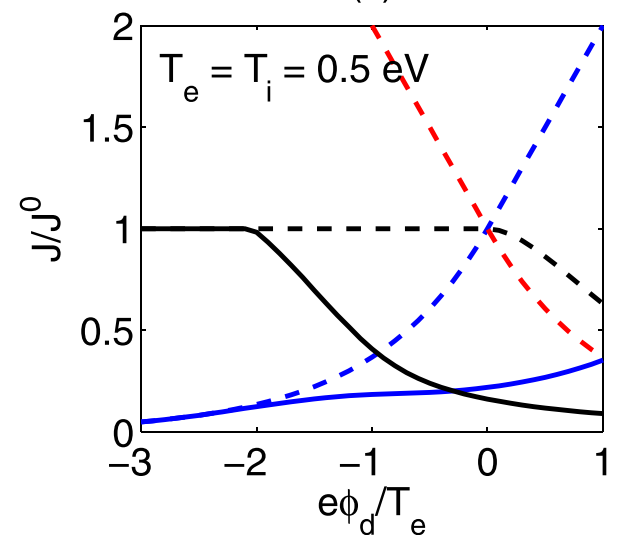

(b)

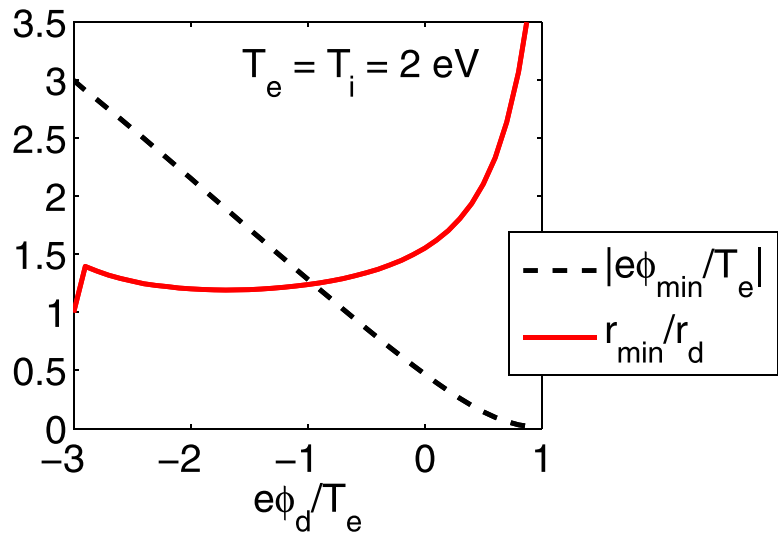

(d)

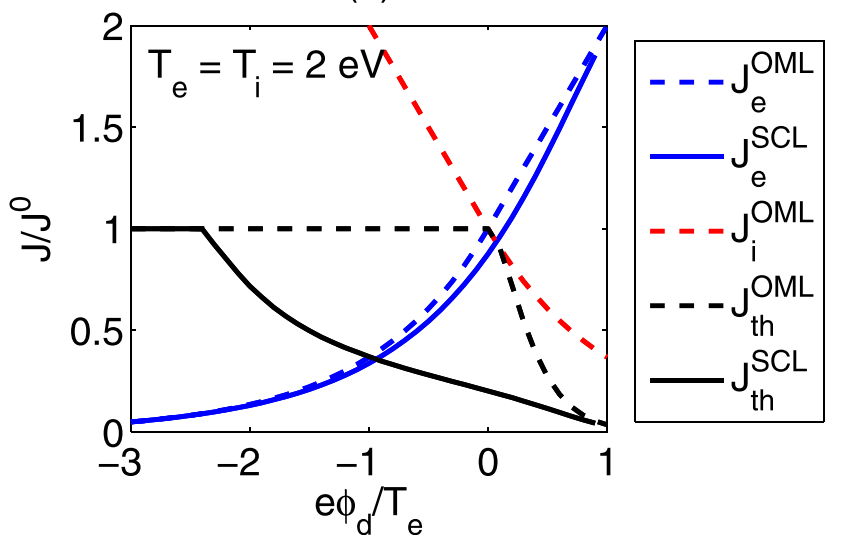

FIG. 8. (a) and (b) VC depth and location from OM radial model approximation and (c) and (d) OML and SCL electron and THE currents and OML ion current against the dust potential. The dust is made of W with radius $r_{d}=1 \mu \mathrm{m}$ and temperature $T_{d}=4500 \mathrm{~K}$. Background plasma parameters are $n_{0}=10^{20} \mathrm{~m}^{-3}$ and $T_{e}=T_{i}=0.5 \mathrm{eV}$ (left) and $T_{e}=T_{i}=2 \mathrm{eV}$ (right)

$J_{\text {th }}^{\mathrm{SCL}}$ starts decreasing as the VC appears and soon becomes significantly lower than $J_{\text {th }}^{\mathrm{OML}}$, though having the same qualitative behavior. This result differs significantly from what one would obtain using the $\mathrm{OML}^{+}$. Indeed, the $\mathrm{OML}^{+}$estimates the VC depth to be lower, resulting in a THE current closer to the OML value (as in Fig. 7).

The primary electron current is also lower in the SCL than in the OML, because the VC acts as a filter that cuts off the low velocity tail of the distribution function. Yet the discrepancy is significant only when $T_{e}$ is of the order of (or lower than) $T_{d}$, i.e., in Fig. 8(c). In Fig. 8(d), for $T_{e}=2 \mathrm{eV}$, primary electrons have, for the most part, enough energy to pass the well (because $T_{e} \gg T_{d}$ ), and the SCL current is very close to the OML result. The much lower electron temperature used in Fig. 8(c) induces a much lower electron current onto the spherical body because most of the impinging electrons bounce back on the VC.

These results are of importance for both dust grains and emissive probes. While the focus of this work is on spherical dust grains, expressions for cylindrical and planar collectors can easily be derived.

\section{DUST ELECTRIC CHARGE IN THE THICK SHEATH REGIME}

The dust particle electric charge $Q_{d}$ is among the most important dust parameters since it dictates particle transport in the plasma via the Lorentz forces, as well as others (ion and electron drag, thermal, etc.). The charge is related to the electric potential through Gauss's law

$$
Q_{d}=\epsilon_{0} \oint_{S_{d}} \nabla \phi . \mathbf{d S},
$$

where $S_{d}$ is the dust surface area. This expression simplifies to $Q_{d}=-4 \pi r_{d}^{2} \epsilon_{0} \phi^{\prime}\left(r_{d}\right)$ in our case. Using a Yukawa potential profile, one obtains ${ }^{25}$

$$
Q_{d}=4 \pi \epsilon_{0} r_{d} \phi_{d}\left(1+\frac{r_{d}}{\lambda_{D}}\right) .
$$

In the thick sheath regime (i.e., when $r_{d} \ll \lambda_{D}$ ), Eq. (37) becomes the equation mainly used in OML-based dust transport codes, $Q_{d}^{\mathrm{OML}}=4 \pi \epsilon_{0} r_{d} \phi_{d}$. As pointed out in Ref. 20, in the SCL regime, $Q_{d}$ can no longer be obtained from this classical expression since the dust electric charge can be positive even whilst the dust potential is negative. Hence, there is a need for a new expression for $Q_{d}$.

Using the double Yukawa profile in Eq. (5), along with $\phi\left(r_{\min }\right)=\phi_{\min }$ and $\phi^{\prime}\left(r_{\min }\right)=0$, we find

$$
\begin{aligned}
\phi^{\prime}\left(r_{d}\right)= & -\frac{\phi_{d}}{r_{d}}\left(1+\xi \frac{r_{d}}{\lambda}\right) \\
& +\frac{\phi_{\min }}{r_{d}}\left(1+\xi \frac{r_{\min }}{\lambda}\right) \exp \left(\frac{r_{\min }-r_{d}}{\lambda}\right) .
\end{aligned}
$$


This expression can be conveniently simplified if we place ourselves in the thick sheath regime, $r_{d}, r_{\min } \ll \lambda / \xi$. In this case

$$
Q_{d}^{\mathrm{SCL}}=4 \pi \epsilon_{0} r_{d} \phi_{d}\left[1-\frac{\phi_{\min }}{\phi_{d}} \exp \left(\frac{r_{\min }-r_{d}}{\lambda}\right)\right] .
$$

The calculated charge $Q_{d}^{\mathrm{SCL}}$ is plotted in Fig. 9 along with the OML result for a W dust grain with $r_{d}=0.1 \mu \mathrm{m}$, $T_{d}=6000 \mathrm{~K}$ and $e \phi_{d} / T_{e}=-0.01$. We used Eqs. (32) and (35) to estimate the values of $r_{\min }$ and $\phi_{\min }$ and assimilated $\lambda$ to the Debye length $\lambda_{D}$.

As expected, the charge sign is changed from the OML result. Moreover, the presence of the VC induces a much higher electric field at the dust surface, leading to a charge more than two times higher in the SCL regime. This could drastically alter the dust transport in tokamak vacuum vessels, since the electric force is directly proportional to $Q_{d}$, while the ion and electron drag forces are proportional to $Q_{d}^{2}$.

\section{CONCLUSION}

New expressions for the collection and emission of electrons by a spherical body in the SCL regime have been derived. They are based on the assumption that the barrier in the effective potential energy is located close to the VC. These expressions can be applied to any type of strongly emissive spherical body immersed in a weakly or nonmagnetized and collisionless plasma. The thick sheath assumption made in the OML theory is no longer required for using the new expressions.

In the SCL regime, the current collection is significantly reduced when the primary electron temperature is of the order of (or lower than) the body temperature. The emission current is always strongly reduced due to the presence of the VC because the average energy of the emitted electrons is of the order of the VC depth.

The association of the current expressions presented in this paper and the equations for the VC parameters from

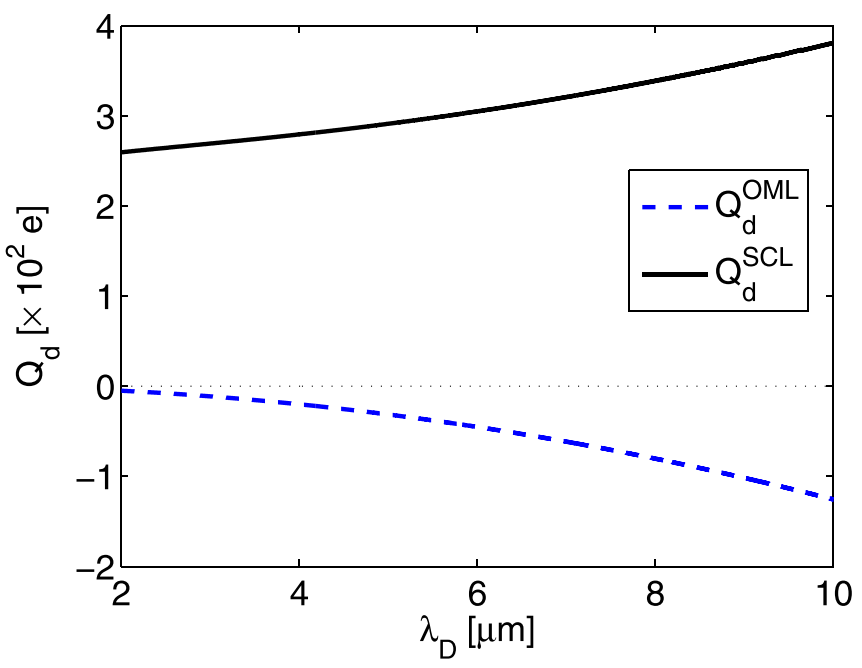

FIG. 9. Dust electric charge against the Debye length in the SCL and OML regimes. A W dust grain with $r_{d}=0.1 \mu \mathrm{m}, T_{d}=6000 \mathrm{~K}$ and $e \phi_{d} / T_{e}=-0.01$ is used in the calculations.
Sec. IV form important progress in comparison with the $\mathrm{OML}^{+}$theory since it is less numerically demanding and the correction to the electron collection current is accounted for.

The determination of the VC parameters (location and depth) remains an important challenge even though some estimates are available.

An expression for the dust electric charge is proposed and can be used when the thick sheath regime applies, which is the case for small grains and/or hot plasmas (since $\lambda_{D} \propto \sqrt{T_{e}}$ ). It leads to changes in the dust charge sign and magnitude that are carried forward to the electric and plasma drag forces a dust grain experiences when transported in a tokamak plasma.

Comparison with experimental data/PIC simulations is planned for future works.

${ }^{1}$ S. I. Krasheninnikov, R. D. Smirnov, and D. L. Rudakov, Plasma Phys. Controlled Fusion 53, 083001 (2011).

${ }^{2}$ J. D. Martin, M. Bacharis, M. Coppins, G. F. Counsell, and J. E. Allen, Europhys. Lett. 83, 65001 (2008).

${ }^{3}$ G. D. Hobbs and J. A. Wesson, Plasma Phys. 9,85 (1967).

${ }^{4}$ S. Takamura, M. Y. Ye, T. Kuwabara, and N. Ohno, Phys. Plasmas 5, 2151 (1998).

${ }^{5}$ L. Dorf, Y. Raitses, and N. J. Fisch, Rev. Sci. Instrum. 75, 1255 (2004).

${ }^{6}$ H. M. Mott-Smith and I. Langmuir, Phys. Rev. 28, 727 (1926).

${ }^{7} \mathrm{~J}$. Laframboise, "The theory of spherical and cylindrical probes is a collisionless, Maxwellian plasma at rest," UTIAS Report No. 100 (University of Toronto Institute for Aerospace Studies, 1966).

${ }^{8}$ J. E. Allen, Phys. Scr. 45, 497 (1992).

${ }^{9}$ R. D. Smirnov, A. Y. Pigarov, M. Rosenberg, S. I. Krasheninnikov, and D. A. Mendis, Plasma Phys. Controlled Fusion 49, 347 (2007).

${ }^{10}$ M. Bacharis, M. Coppins, and J. E. Allen, Phys. Plasmas 17, 042505 (2010).

${ }^{11}$ E. Lazzaro, I. Proverbio, F. Nespoli, S. Ratynskaia, C. Castaldo, U. deAngelis, M. DeAngeli, J.-P. Banon, and L. Vignitchouk, Plasma Phys. Controlled Fusion 54, 124043 (2012).

${ }^{12}$ L. Vignitchouk, P. Tolias, and S. Ratynskaia, Plasma Phys. Controlled Fusion 56, 095005 (2014).

${ }^{13}$ A. Autricque, S. Hong, N. Fedorczak, S. Son, H. Lee, I. Song, W. Choe, and C. Grisolia, in Proceedings of the 22nd International Conference on Plasma Surface Interactions, 2016 [Nucl. Mater. Energy 12, 599 (2017)].

${ }^{14}$ M. Y. Ye and S. Takamura, Phys. Plasmas 7, 3457 (2000).

${ }^{15}$ S. Takamura, N. Ohno, M. Y. Ye, and T. Kuwabara, Contrib. Plasma Phys. 44, 126 (2004).

${ }^{16}$ A. Din, Phys. Plasmas 20, 093505 (2013).

${ }^{17}$ F. Taccogna, Eur. Phys. J. D 68, 199 (2014).

${ }^{18}$ J. P. Sheehan and N. Hershkowitz, Plasma Sources Sci. Technol. 20, 063001 (2011).

${ }^{19}$ A. Fruchtman, D. Zoler, and G. Makrinich, Phys. Rev. E 84, 025402 (2011).

${ }^{20}$ G. L. Delzanno and X.-Z. Tang, Phys. Rev. Lett. 113, 035002 (2014).

${ }^{21}$ X.-Z. Tang and G. L. Delzanno, Phys. Plasmas 21, 123708 (2014).

${ }^{22}$ M. D. Campanell and M. V. Umansky, Phys. Rev. Lett. 116, 085003 (2016).

${ }^{23}$ M. D. Campanell and M. V. Umansky, Phys. Plasmas 24, 057101 (2017).

${ }^{24}$ B. F. Kraus and Y. Raitses, Phys. Plasmas 25, 030701 (2018).

${ }^{25}$ E. C. Whipple, Rep. Prog. Phys. 44, 1197 (1981).

${ }^{26}$ Y. L. Al'pert, A. V. Gurevich, and L. P. Pitaevskii, Space Physics with Artificial Satellites (Consultants Bureau, New York, 1965).

${ }^{27}$ V. Fortov, A. Ivlev, S. Khrapak, A. Khrapak, and G. Morfill, Phys. Rep. 421, 1 (2005).

${ }^{28}$ I. B. Bernstein and I. N. Rabinowitz, Phys. Fluids 2, 112 (1959).

${ }^{29}$ J. E. Allen, B. M. Annaratone, and U. de Angelis, J. Plasma Phys. 63, 299-309 (2000)

${ }^{30}$ R. V. Kennedy and J. E. Allen, J. Plasma Phys. 69, 485-506 (2003).

${ }^{31}$ G. L. Delzanno and G. Lapenta, Phys. Rev. Lett. 94, 175005 (2005).

${ }^{32}$ G. L. Delzanno, A. Bruno, G. Sorasio, and G. Lapenta, Phys. Plasmas 12, 062102 (2005)

${ }^{33}$ S. Dushman, Rev. Mod. Phys. 2, 381 (1930).

${ }^{34}$ M. S. Sodha, J. Appl. Phys. 32, 2059 (1961).

${ }^{35}$ J. Martin, "Theory and simulation of dust in tokamak plasmas," Ph.D. thesis (Imperial College London, 2006). 